PURD-TH-94-01

VAND-TH-94-01

\title{
MASS BOUNDS IN THE STANDARD MODEL
}

\author{
T.E. Clark, B. Haeri, S.T. Love, M.A. Walker \\ Department of Physics \\ Purdue University \\ West Lafayette, IN 47907-1396 \\ and \\ W.T.A. ter Veldhuis \\ Department of Physics and Astronomy \\ Vanderbilt University \\ Nashville, TN 37235
}

\begin{abstract}
Nonperturbative triviality and vacuum stability mass bounds are obtained for the Higgs scalar and top quark degrees of freedom in the standard electroweak model using Wilson renormalization group techniques. Particular attention is given to the effect of the generalized top Yukawa coupling on the scalar mass upper bound.
\end{abstract}


The scalar sector of the standard electroweak model which is responsible for the electroweak symmetry breaking is characterized by two parameters. The dimensionful scalar mass term coupling is phenomenologically set by the electroweak symmetry breaking scale, while the dimensionless quartic scalar self coupling is related to the magnitude of the physical Higgs scalar mass. While this mass is completely unconstrained at tree level, the radiative corrections lead to inconsistencies unless the mass is constrained to be less than some upper bound. This so-called triviality bound is a reflection of the infrared Gaussian (trivial) fixed point of the model which, in turn, can be traced to the non-asymptotically free nature of the scalar self coupling. Its increasing growth tends to destabilize the ultraviolet physics unless suitably constrained.

The triviality bound has been calculated in perturbation theory ${ }^{[1]}$ and found to produce a scalar mass upper bound of $\mathrm{O}(1 \mathrm{TeV})$. However, since such a scalar mass corresponds to a nonperturbative value of the scalar self coupling, the result requires further scrutiny via calculation within a nonperturbative framework. Various nonperturbative studies ranging from lattice simulations $^{[2]}$ to $1 / \mathrm{N}$ expansions ${ }^{[3]}$ have been carried out for the self coupled scalar system. Another approach advocated by Hasenfratz and Nager ${ }^{[4]}$ employs a nonperturbative Wilson renormalization group equation ${ }^{[5]}$. In each case, the perturbative result has been substantiated and an upper bound Higgs scalar mass of $\mathrm{O}(1 \mathrm{TeV})$ has again been obtained. The basic equality of these bounds can be traced to the presence of the infrared Gaussian fixed point in all analyses.

Of course, when considering the standard model, the scalar sector should not be treated in isolation. Since the Higgs mass bound arises from con- 
straints on the ultraviolet physics where the gauge couplings are all small, their effect can be adequately accounted for within perturbation theory. The scalar doublet also couples to the various fermions via Yukawa couplings whose magnitude is set by the associated fermion mass. Since these couplings are also non-asymptotically free, the self consistency of the model likewise forces them to be appropriately constrained. All the presently observed fermions are sufficiently light that the effects of their Yukawa coupling are also amenable to a perturbative study. On the other hand, the present experimental bound on the top quark mass is such that its Yukawa coupling may require a nonperturbative analysis.

In the present note, we determine the allowed range of Higgs scalar and top quark masses using a Wilson renormalization group equation (WRGE) approach. Such an equation containing both scalars and fermions was obtained previously ${ }^{[6]}$ by three of the authors and can be applied here. The WRGE nonperturbatively relates the form of the Euclidean action at a scale $\Lambda(t)=e^{-t} \Lambda$ to the action at scale $\Lambda$ for $t>0$. It is derived by demanding that the physics, ie. correlation functions, remain unchanged as degrees of freedom carrying momentum between the scales $\Lambda$ and $e^{-t} \Lambda$ are integrated out. Thus either action can be used to equivalently describe the physics on all scales less than $e^{-t} \Lambda$ and both actions lie on the same Wilson renormalization group trajectory. The lower scale action is constructed by appropriately changing the coefficients of the operators already present in the scale $\Lambda$ action and by introducing new operators which were not present at scale $\Lambda$ in such a way so as to keep the physics unchanged.

In our previous work ${ }^{[6]}$, we applied the equation to study a Higgs-Yukawa model of a Dirac fermion interacting with a real scalar in a way which was 
invariant under a discrete $\gamma_{5}$ symmetry which prevented an explicit fermion mass term. Since one of the major attributes of the formalism is that it can also be used to describe chiral fermions, we can also employ the same equation to study the standard model which contains chiral fermions interacting with a complex electroweak doublet of scalar fields in a $S U(2)_{L} \times U(1)$ invariant manner. Since the gauge interactions can be handled perturbatively, we do not have to use the full WRGE structure in their treatment. This is fortuitous since the WRGE formalism is not easily adaptable to deal with strong gauge couplings. Unfortunately, just as in the discrete symmetry case, to render the WRGE tractable to analysis even in the absence of gauge interactions requires the introduction of various approximations. The first of these is a local action approximation ${ }^{[4,6]}$ which neglects anomalous dimensions and derivative interactions and thus constitutes a first term in a momentum expansion. The WRGE then reduces to an equation for a generalized potential function $U(\rho, Y ; t)$, where $\rho=2 \varphi^{\dagger} \varphi$ and $Y=\sqrt{2}\left(\bar{t}_{L} t_{R} \varphi_{0}+\bar{b}_{l} t_{R} \varphi_{-}+\bar{t}_{R} b_{L} \varphi_{+}+\bar{t}_{R} t_{L} \varphi_{0}^{\dagger}\right)$. Here we have assumed that all the fermion dependence of $U$ appears as a function of $Y$ which is the $S U(2) \times U(1)$ invariant coupling of the scalar doublet $\phi^{\dagger}=\left(\varphi_{0}^{\dagger}, \varphi_{+}\right)$and its conjugate to the top and bottom quarks, $t, b$, respectively. So doing, we have neglected possible dependences on the fermions appearing in higher dimensional (eg. four-fermion) $S U(2) \times U(1)$ invariant structures. A further truncation, made solely to facilitate the subsequent numerical analysis, is to retain terms in $U$ only up to linear in $Y$, but still multiplying a general function of $\rho$. We thus write

$$
U(\rho, Y ; t)=V(\rho, t)+Y G(\rho, t)
$$

as the sum of an effective potential $V(\rho, t)$ which determines the vacuum structure and a generalized Yukawa interaction $Y G(\rho, t)$. Defining $F(\rho, t)=$ 
$V_{\rho}(\rho, t)=\frac{\partial V(\rho, t)}{\partial \rho}$, (subscripts denoting differentiation) so that the ground state corresponds to the zeroes of $F$, the WRGE ${ }^{[7]}$ reduces to the two coupled partial differential equations

$$
\begin{aligned}
\frac{\partial F}{\partial t}=\frac{1}{16 \pi^{2}} & {\left[\frac{6 F_{\rho}}{1+2 F}+\frac{6 F_{\rho}+4 \rho F_{\rho \rho}}{1+2 F+4 \rho F_{\rho}}-\frac{12 G^{2}+24 \rho G G_{\rho}}{1+\rho G^{2}}\right]+2 F-2 \rho F_{\rho} } \\
\frac{\partial G}{\partial t}=\frac{1}{16 \pi^{2}} & {\left[\frac{6 G_{\rho}}{1+2 F}+\frac{6 G_{\rho}+4 \rho G_{\rho \rho}}{1+2 F+4 \rho F_{\rho}}\right.} \\
& \left.+8 \rho G\left[\frac{G^{2} F_{\rho}-(1+2 F)\left(G+\rho G_{\rho}\right) G_{\rho}}{\left(1+\rho G^{2}\right)\left(1+2 F+4 \rho F_{\rho}\right)(1+2 F)}\right]\right]-2 \rho G_{\rho} .
\end{aligned}
$$

It is straightforward to check that these equations reproduce the perturbative running of the ordinary Yukawa and scalar quartic coupling in the absence of anomalous dimensions.

To proceed with the analysis, the WRGE must be supplemented with the form of the generalized potential at some initial scale $\Lambda=\Lambda(t=0)$. Choosing the $S U(2)_{L} \times U(1)$ invariant standard model form

$$
U(\rho, Y ; t=0)=\frac{1}{2} m^{2}(0) \rho+\frac{1}{4} \lambda(0) \rho^{2}+\frac{1}{\sqrt{2}} g_{t}(0) Y,
$$

we then seek solutions for $F$ and $G$ which spontaneously break this symmetry, ie. $\left\langle\varphi_{0}\right\rangle \neq 0$. This in turn restricts the $m^{2}(0), \lambda(0), g_{t}(0)$ parameter space. To implement the restriction, we define $m_{c r}^{2}$ as the maximum value of $m^{2}(0)$ for a given $\lambda(0), g_{t}(0)$ which results in a nontrivial zero of $F(\rho, t)$ as $t$ increases into the infrared. $m_{c r}^{2}$ is determined by evaluating $F(\rho, t)$ for $m^{2}(0)$ well into the broken phase and then increasing $m^{2}(0)$ until the zero of $F$ decreases as $t$ increases. The value of $m^{2}(0)$ which produces this transition so that the only zero of $F$ as $t \rightarrow \infty$ is at the origin defines $m_{c r}^{2}$. So doing, we establish the infrared Gaussian fixed point. From the solution, we also see that the induced irrelevant operators can give sizeable contributions for small $t$ values 
and play an important role in driving the theory toward this fixed point. Furthermore, the numerically generated solution to the WRGE is seen to smoothly join onto the 1-loop solution (which includes irrelevant operators) for $t$ values beyond some $t^{*}$.

To extract the Higgs scalar and top quark mass bounds, we choose a point in the allowed $\lambda(0), g_{t}(0), m^{2}(0)<m_{c r}^{2}$ parameter space corresponding to the spontaneous symmetry breaking solution and then numerically integrate the WRGE to obtain $F$ and $G$ for $0<t<t^{*}$. We next include the degrees of freedom with $|p|<e^{-t^{*}} \Lambda$ via the 1-loop perturbative solution which smoothly joins onto the numerically generated solution at $t^{*}$. As a consequence of the spontaneous symmetry breakdown, the model spectrum also contains three Nambu-Goldstone degrees of freedom which upon gauging the $S U(2) \times U(1)$ symmetry become, via the Higgs mechanism, the longitudnal components of the $W_{ \pm}, Z$ vector bosons. Due to the presence of these massless modes, care must be exhibited in integrating all the way down into the infrared. Following the procedure used in the pure scalar model ${ }^{[4]}$, we simply cut off the 1-loop integrals in the infrared at a scale $p^{2}=M_{W}^{2}$. The computed masses turn out to be only weakly dependent on the choice of the infrared cutoff. Summing the contributions produces the functions $F^{e f f}(\rho)$ and $G^{e f f}(\rho)$ from which the nontrivial scalar vacuum expectation value $(v)$ and Higgs scalar and top quark masses $\left(M_{H}\right.$ and $m_{t}$ respectively) are secured as

$$
\begin{aligned}
F^{e f f}\left(\frac{v^{2}}{\Lambda^{2}}\right) & =0 \\
\frac{M_{H}^{2}}{\Lambda^{2}} & =4 \frac{v^{2}}{\Lambda^{2}} F_{\rho}^{e f f}\left(\frac{v^{2}}{\Lambda^{2}}\right) \\
\frac{m_{t}}{\Lambda} & =\left.G^{e f f}\left(\frac{v^{2}}{\Lambda^{2}}\right) \frac{\partial Y}{\partial(\bar{t} t)}\right|_{\varphi_{0}=\varphi_{0}^{\dagger}=v /(\sqrt{2} \Lambda)} .
\end{aligned}
$$




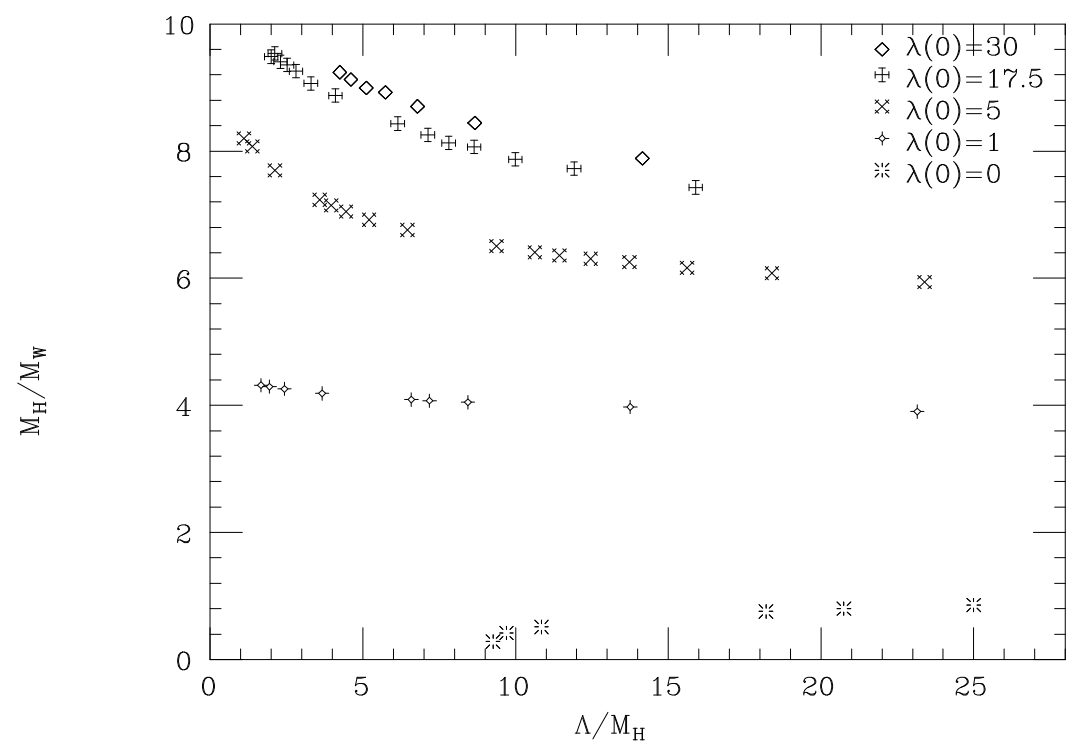

Figure 1: $M_{H} / M_{W}$ as a function of $\Lambda / M_{W}$ for various $\lambda(0)$ values and

$$
g_{t}(0)=0.6
$$

The value of $t^{*}$ is chosen such that the computed masses are stable to within an error the size of the $t$-grid spacing: $10^{-5}<\delta t<10^{-4}$. Finally, the computation is repeated varying over the allowed parameter space.

Using the above procedure, we extract the ratios $M_{H} / \Lambda, m_{t} / \Lambda$ and $v / \Lambda$ as the couplings $\lambda(0)$ and $g_{t}(0)$ are varied. Taking various products of these ratios and using the relation between $v \simeq 246 \mathrm{GeV}$ and the $W_{ \pm}$mass, we plot in Figures 1 and 2 the allowed $M_{H} / M_{W}$ values as a function of $\Lambda / M_{H}$ for a range of initial $\lambda(0)$ values and $g_{t}(0)=0.6$ and 2.0 respectively. The $g_{t}(0)=$ 0.6 plot is virtually identical (to within the error bars) to the analogous $g_{t}(0)=0 \operatorname{plot}^{[4]}$. 


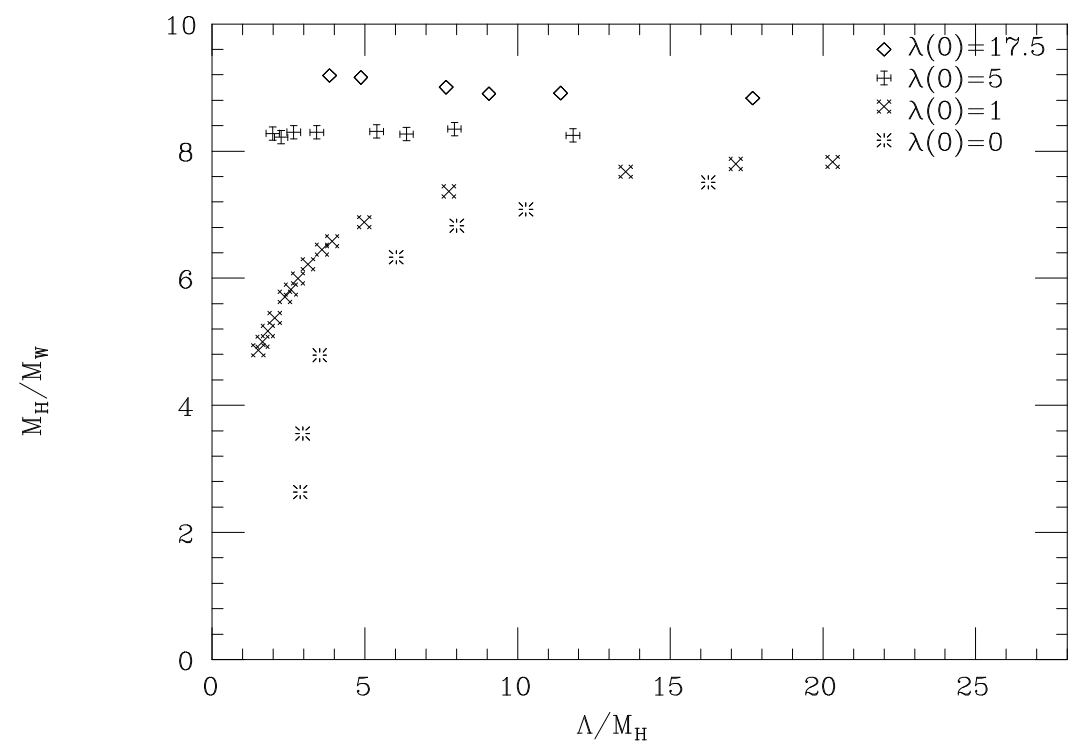

Figure 2: $M_{H} / M_{W}$ as a function of $\Lambda / M_{W}$ for various $\lambda(0)$ values and

$$
g_{t}(0)=2.0 \text {. }
$$

From the plotted data, we see in both cases that a dynamical envelope is formed as the initial $\lambda(0)$ coupling is varied. That is, for fixed $\Lambda / M_{W}$, the different $M_{H} / M_{W}$ values appear to converge to an upper limit. When this is combined with the physical requirement that no mass is allowed to become larger than the initial cutoff $\Lambda$ so that $\Lambda / M_{H}>1$, we secure an upper bound on the allowed $M_{H} / M_{W}$ ratio of roughly 10 which corresponds to the bound $M_{H} \leq 800 \mathrm{GeV}$. This is consistent with the results obtained using lattice simulations ${ }^{[7]}$ as well as with the $1 / \mathrm{N}^{[8]}$ calculations. Note that changing $g_{t}(0)$ from 0.6 to 2.0 produces but a small increase in the Higgs scalar mass upper bound.

We can also use the data to extract the allowed domain of scalar Higgs and top quark masses at a fixed cutoff to $M_{W}$ ratio. Figure 3 is just such 


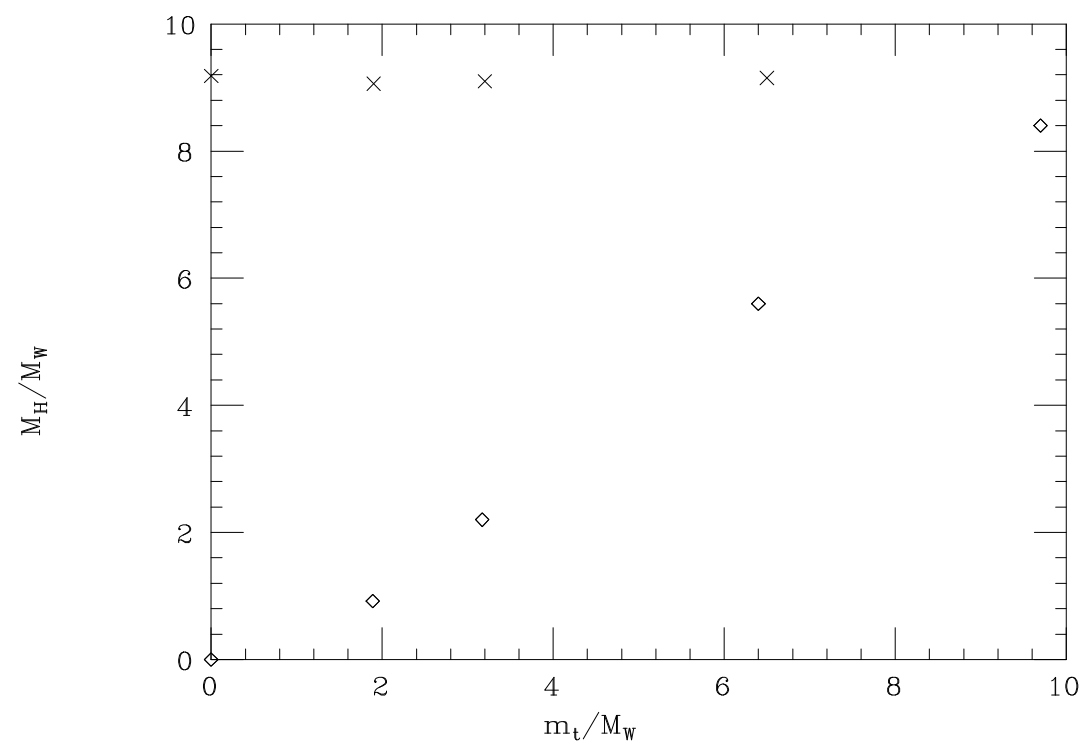

Figure 3: The allowed range of $M_{H} / M_{W}$ and $m_{t} / M_{W}$ values for

$$
\Lambda / M_{W}=20 .
$$

a plot corresponding to $\Lambda / M_{W}=20$. The allowed values are those lying interior to the various boundaries displayed. The upper portion of boundary, denoted by the cross $(\times)$ marks is the scalar triviality bound discussed above. The flatness of this curve as a function of the top quark mass testifies to the insensitivity of the Higgs scalar mass upper bound to the top Yukawa coupling. The points on the figure represented by the diamond $(\diamond)$ markings arise from the vacuum stability requirement which corresponds to the condition that $\lim _{|\rho| \rightarrow \infty} F(\rho, t)>0$ for all $t$. Note that for $m_{t} \simeq 200$ $\mathrm{GeV}$, the lower bound on $M_{H}$ is around $60 \mathrm{GeV}$ which is roughly the current experimental lower bound.

We have used the Wilson renormalization group equation to construct nonperturbative mass bounds on the Higgs scalar and top quark degrees of 
freedom in the electroweak standard model. We found a Higgs scalar mass absolute upper bound of approximately $800 \mathrm{GeV}$ which was quite insensitive to the presence of the top quark Yukawa coupling and thus basically identical to the WRGE calculation in the pure $O(4)$ scalar mode ${ }^{[4]}$ and a model with an additional scalar singlet ${ }^{[9]}$. Our results are also consistent with other mass bound estimates based on lattice simulations. Of course, our study is subject to the various uncertainties which accompany the approximation schemes. In particular, it is desirable to extend our analysis beyond the local approximation to include non-trivial anomalous dimensions and derivative interactions. In addition, one should also include the effects of the gauge interactions and the light quark Yukawa couplings. Since these effects can be treated perturbatively ${ }^{[10]}$ and are small we do not include them here.

\section{Acknowledgements}

The work of T.E.C., B.H. and S.T.L. was supported in part by the U.S. Department of Energy under grant DE-ACO2-76ER01428 (Task B). The work of W.T.A.t.V. was supported by the U.S. Department of Energy under grant DE-FG05-8SER40226. 


\section{References}

1. R. Dashen and H. Neuberger, Phys. Rev. Lett. 50 (1983) 1897; M.A.B. Beg et al, Phys. Rev. Lett. 52 (1984) 833; D.J.E. Callaway, Nucl. Phys. B223 (1984) 189; M. Lindner, Z. Phys. C31 (1986) 295.

2. M. Lüscher and P. Weisz, Phys. Lett. 212B (1988) 472; Nucl. Phys. B318 (1989) 705; J. Kuti, L. Lin and Y. Shen, Phys. Rev. Lett. 61 (1988) 678; G. Bhanot, K. Bitar, U. Heller and H. Neuberger, Nucl. Phys. B353 (1991) 551.

3. M. Einhorn, Nucl. Phys. B246 (1984) 75.

4. P. Hasenfratz and J. Nager, Z. Phys.C37 (1988) 477.

5. K.G. Wilson and J.B. Kogut, Phys. Rept. 12C (1974) 75; K.G. Wilson, Rev. Mod Phys. 55 (1983) 583; F.J. Wegner and A. Houghton, Phys. Rev. A8 (1973) 401; S. Weinberg, in Proceedings of the 1976 International School of Subnuclear Physics, Erice, ed. by A. Zichichi (Plenum Press, 1978); see also J. Polchinski, Nucl. Phys. B231 (1984) 269; C. Wetterich, Nucl. Phys. B352 (1991) 529; U. Ellwanger and L. Vergara, Nucl. Phys. B398 (1993) 52; T. Morris, preprint CERN-TH.6977/93.

6. T.E. Clark, B. Haeri and S.T. Love, Nucl. Phys B402 (1993) 628.

7. Y. Shen, J. Kuti, L. Lin and P. Rossi, Nucl. Phys. (Proc. Suppl.) 9 (1989) 99.

8. M. Einhorn and G. Goldberg, Phys. Rev. Lett. 57 (1986) 2115; G. Bathas and H. Neuberger, Nucl. Phys. (Proc. Suppl.) 30 (1993) 635. 
9. R. Akhoury and B. Haeri, Phys. Rev. D48 (1993) 1252.

10. L. Maiani, G. Parisi and R. Petronzio, Nucl. Phys. B136 (1978) 115;

N. Cabibbo et al, Nucl. Phys. B158 (1979) 295. 